\title{
Renal salt wasting syndrome in a patient with COVID-19; a case report and review of the literature
}

\author{
Bassam Al-Helal $^{1 \oplus}$, Emad Abdallah $^{1 * \oplus}$, Altayyeb Yousef $^{2}$, Reem Asad $^{1 \oplus}$, Mahmoud Reda $^{1}$
}

\begin{abstract}
Introduction: Cerebral salt wasting or renal salt wasting (RSW) syndrome, may be more common than syndrome of inappropriate antidiuretic hormone secretion (SIADH) and may even occur in the absence of cerebral disease. We report a case of RSW in a Bangladeshi patient positive for COVID-19 without clinical cerebral disease.

Case Presentation: A 53 years-old Bangladeshi patient presented with history of chest pain and acute MI. On examination, the patient was conscious, alert, vitally stable, chest with fine bilateral basal crepitation and heart with additional S3 sound and abdomen was lax with no organomegaly. There was no lower limbs oedema. His serum creatinine; $68 \mathrm{umol} / \mathrm{L}$, urea; $3.4 \mathrm{mmol} / \mathrm{L}, \mathrm{K} ; 4.7 \mathrm{mmol} / \mathrm{L}$, sodium; 135 $\mathrm{mmol} / \mathrm{L}$, uric acid; $141 \mathrm{mmol} / \mathrm{L}$ and phosphate was $1.3 \mathrm{mmol} . / \mathrm{L}$. Echocardiography (ECG) revealed anterior lateral wall STEMI. PCI was done for LAD. ECG revealed ejection fraction (EF) 10-15\%. Nasopharyngeal swab for COVID-19 was positive. Serum sodium decreased from 135 to 108 with signs of hypovolemia. Work up for hyponatremia revealed serum osmolality of $237 \mathrm{mOsm} / \mathrm{kg}$, urine NA; 109 $\mathrm{mmol} / \mathrm{L}$, urine osmolality; $295 \mathrm{mOsm} / \mathrm{kg}$, urine uric acid; $685 \mathrm{umol} / \mathrm{L}$, and urine phosphate; $6.5 \mathrm{mmol} / \mathrm{L}$. Additionally serum T3, T4, TSH and serum basal cortisol were normal. The patient received normal saline infusion and fludrocortisone and serum sodium increased to 134 $\mathrm{mmol} / \mathrm{L}$. Our patient had all the important clinical and laboratory characteristics of RSW in the absence of cerebral disease which include hyponatremia associated with hypovolemia, high urinary sodium excretion, increased fraction excretion of phosphate and persistent hypouricemia with increased fractional excretion of urate after correction of hyponatremia and with normal renal, adrenal and thyroid functions. Furthermore, there was a prompt response to saline replacement and fludrocortisone and steady improvement in serum sodium with negativity and improvement of COVID-19. Our diagnosis was RSW in the absence of cerebral disease and to our knowledge; this is the first case of RSW in a patient with COVID-19 in the literature.

Conclusion: RSW should be considered in patients with COVID-19 with hyponatremia and absence of cerebral disease. We suggest changing cerebral salt wasting to the more appropriate term RSW.

Keywords: COVID-19, Cerebral salt-wasting syndrome, Renal salt wasting syndrome, Syndrome of inappropriate antidiuretic hormone secretion, SARS-CoV-2

Citation: Al-Helal B, Abdallah E, Yousef A, Asad R, Reda M. Renal salt wasting syndrome in a patient with COVID-19; a case report and review of the literature. J Renal Endocrinol. 2021;7:e02. doi: 10.34172/jre.2021.02.

Copyright $\odot 2021$ The Author(s); Published by Nickan Research Institute. This is an open-access article distributed under the terms of the Creative Commons Attribution License (http://creativecommons.org/licenses/by/4.0), which permits unrestricted use, distribution, and reproduction in any medium, provided the original work is properly cited.
\end{abstract}

\section{Introduction}

Cerebral salt-wasting syndrome (CSW), or renal salt wasting (RSW) described by Peters et al in 1950 (1), and may be more common than syndrome of inappropriate antidiuretic hormone secretion (SIADH). CSW syndrome is defined by the development of extracellular volume depletion due to a renal sodium transport abnormality in patients with intracranial disease and normal adrenal and thyroid function $(1,2)$. CSW may even occur in the absence of cerebral disease (3-5). As such, it may be more appropriately termed RSW syndrome.

COVID-19 - the disease caused by the coronavirus that's led to the global pandemic - is known to damage the lungs. But, as more people become infected, more understanding of the disease emerges. Researchers are finding that this coronavirus - officially called SARS-CoV-2-can also cause severe and lasting harm in other organs, including the heart and kidneys (6-12). We report a case of RSW syndrome in a Bangladeshi patient positive for COVID-19 and to our knowledge; this is the first case report of RSW in a patient with COVID-19 in the literature.

\section{Case Presentation}

A 53 years-old Bangladeshi patient presented to AlAdan hospital casualty, Kuwait on 28/4/2020, with a history of chest pain and acute myocardial infarction. On examination, the patient was conscious, alert, body mass index was $30 \mathrm{~kg} / \mathrm{m}^{2}$, blood pressure $113 / 57 \mathrm{~mm}$ $\mathrm{Hg}$, pulse $84 \mathrm{~b} / \mathrm{m}$, temperature $37.3^{\circ} \mathrm{C}$, chest with fine bilateral basal crepitation and heart with additional S3 sound and abdomen was lax with no organomegaly. There was no lower limbs edema. His serum creatinine ;68 umol/L, urea; $3.4 \mathrm{mmol} / \mathrm{L}, \mathrm{K} ; 4.7 \mathrm{mmol} / \mathrm{L}$, sodium; $130 \mathrm{mmol} / \mathrm{L}$, uric acid; $141 \mathrm{mmol} / \mathrm{L}, \mathrm{ALT} ; 39 \mathrm{IU} / \mathrm{L}, \mathrm{AST}$; 
$30 \mathrm{IU} / \mathrm{L}$ and serum albumin of $36.1 \mathrm{~g} / \mathrm{L}$. Serum calcium; $2.4 \mathrm{mmol} / \mathrm{L}$ and phosphorus was $1.3 \mathrm{mmol} / \mathrm{L}$. CBC revealed hemoglobin of $10.3 \mathrm{~g} / \mathrm{dL}$, WBCs $3.4 \times 10^{9} / \mathrm{L}$ with a normal differential and platelet count of $174 \times 10^{\%} / \mathrm{L}$. Lipid profiles revealed total cholesterol $6.34 \mathrm{mmol} / \mathrm{L}$, LDL-cholesterol $3.84 \mathrm{mmol} / \mathrm{L}$, HDL-cholesterol 1.12 $\mathrm{mmol} / \mathrm{L}$ and triglycerides $2.17 \mathrm{mmol} / \mathrm{L}$. Urine analysis revealed WBCs 6-10/hpf, RBCs 61-80/hpf, urine PH 7, no casts with good urine output $70-80 \mathrm{~mL} / \mathrm{h}$. ECG revealed anterior lateral wall STEMI. PCI was done (29/4/2020) for LAD with IABP for cardiogenic shock which was removed after 24 hours. Echocardiography revealed EF 10-15\%. Nasopharyngeal swab for COVID-19 (11/5/2020) was positive. Serum sodium decreased from 135 (28/4/2020) to $108(11 / 5 / 2020)$ with signs of hypovolaemia. Work up for hyponatremia revealed serum osmolality $237 \mathrm{mOsm} /$ $\mathrm{kg}$, urine sodium; $109 \mathrm{mmol} / \mathrm{L}$, urine $\mathrm{K} ; 11.5 \mathrm{mmol} / \mathrm{L}$, urine osmolality $295 \mathrm{mOsm} / \mathrm{kg}$, urine CL $104 \mathrm{mmol} / \mathrm{L}$, urine uric acid $685 \mathrm{umol} / \mathrm{L}$, urine creatinine $1.9 \mathrm{mmol} / \mathrm{L}$, urine urea $49 \mathrm{mmol} / \mathrm{L}$ and urine phosphate $6.5 \mathrm{mmol} / \mathrm{L}$. Serum T3, T4, TSH and serum basal cortisol were normal. Serum PCT; 0.27, CRP; 53, PH; 7.4, HCO3; 20.3 and PCO2 was 3.9. The patient was on lasix and spironolactone for one day and stopped when the patient developed hyponatremia and this stopping did not lead to correction of hyponatremia.

The patient received normal saline infusion $200 \mathrm{~mL} / \mathrm{h}$ and serum sodium increased to $131 \mathrm{mmol} / \mathrm{L}$ and then dropped again to $124 \mathrm{mmol} / \mathrm{L}$ and fludrocortisone $0.2 \mathrm{mg}$ tab twice daily (only two doses were given) was initiated with normal saline $60 \mathrm{~mL} / \mathrm{h}$ and serum sodium increased to $128-129 \mathrm{mmol} / \mathrm{L}$ and normal saline stopped when serum sodium became 132 on May 22, 2020 and with follow up serum sodium was 134 on May 30, 2020 (Table 1). Serum electrolytes, BUN, creatinine, urate, and phosphate were determined concomitantly with urine osmolality, sodium, potassium, creatinine, urate, and phosphate at regular intervals during the study. Plasma renine, aldosterone, $\mathrm{ADH}$ not were assessed. Our patient had all the important clinical and laboratory characteristic of RSW in the absence of cerebral disease including hyponatremia associated with hypovolemia, high urinary sodium excretion, increased fraction excretion of phosphate (FE phosphate) and persistent hypouricemia with increased fractional excretion of urate (FE urate) after correction of hyponatremia and with normal renal, adrenal and thyroid functions. Furthermore, there was a prompt response to saline replacement and fludrocortisone and steady improvement in serum sodium with improvement and negativity of COVID-19 (Table 1). Our diagnosis was RSW in the absence of cerebral disease and to our knowledge, this is the first case report of RSW in a patient with COVID-19 in the literature.

\section{Discussion}

Hyponatremia is the most common electrolyte
Implication for health policy/practice/research/ medical education

Renal salt wasting syndrome should be considered in patients with COVID -19 with hyponatremia and absence of cerebral disease.

abnormality that is undergoing dramatic changes in terms of its diagnostic approach and clinical outcomes (13).

In CSW or RSW, abnormalities in the proximal tubule result in excessive sodium losses, which lead to decreased effective circulating volume. This activates baroreceptors, which increase antidiuretic hormone (ADH) secretion. This results in water conservation and a return to an equilibrated state. In contrast, SIADH primarily occurs due to an inappropriate euvolemic rise in $\mathrm{ADH}$ secretion (14). In our case the abnormalities most probably in proximal tubule due to increased urinary sodium excretion with increased FEurate, urea and phosphate.

Differentiation of this disorder from the SIADH, a common cause of hyponatremia, can be difficult because both can present with hyponatremia and concentrated urine with natriuresis. However, distinguishing between the two disorders is important because treatment options differ. Attention to the volume status of the patient is important in making the distinction. Failure to distinguish CSW syndrome from SIADH in a patient with hyponatremia who has brain injury could lead to inappropriate therapy with fluid restriction. RSW should be considered a discrete clinical entity and may be more common than perceived (4). It should also be considered in patients without cerebral disease $(3,15)$.

The relationship among serum urate, FEurate, and hyponatremia in CSW syndrome is unclear. FEurate may remain elevated even after correction of hyponatremia in patients with CSW syndrome. This is distinct from SIADH, in which FEurate returns to the reference range once the hyponatremia is corrected $(4,15)$. The physiologic basis for this in CSW syndrome may be related to the receptormediated processing of sodium and urate in the proximal tubule, which may be defective in this syndrome. The physiologic basis for hypouricemia in SIADH remains unclear.

The abnormalities in proximal tubular transport may be secondary to a plasma natriuretic factor that reduces proximal and, possibly, distal sodium transport in renal salt-wasting syndrome. It may also inhibit the tubular transport of urate, phosphate, and urea in addition to sodium (16).

The cause of kidney involvement in COVID-19 is likely to be multifactorial, with cardiovascular comorbidity and predisposing factors (such as sepsis, hypovolemia, and nephrotoxins) as important contributors (6).

Cardiorenal syndrome, particularly right ventricular failure secondary to COVID-19 pneumonia, might lead 


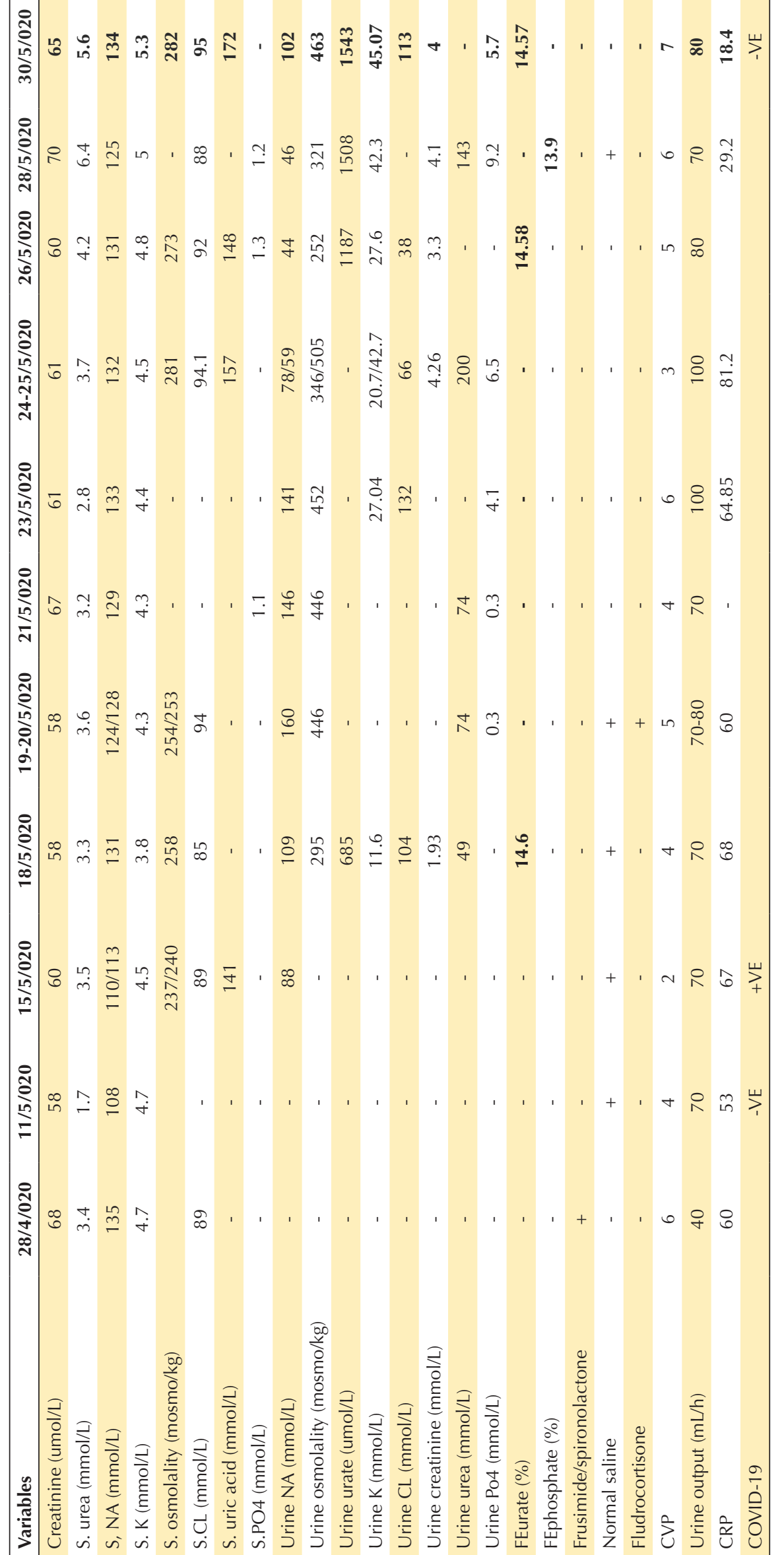


to kidney congestion and subsequent acute kidney injury (AKI). Similarly, left ventricular dysfunction might lead to low cardiac output, arterial under-filling, and kidney hypoperfusion.

Autopsy data (7), indicates that the endothelium is affected in the lung and in the kidney, where it is probably responsible for proteinuria. Furthermore, virus particles were reported to be present in renal endothelial cells, indicating viremia as a possible cause of endothelial damage in the kidney and a probable contributor to AKI (7).

Additionally, SARS-CoV-2 can directly infect the renal tubular epithelium and podocytes through an angiotensinconverting enzyme 2 (ACE2)-dependent pathway and cause mitochondrial dysfunction, acute tubular necrosis, the formation of protein reabsorption vacuoles, collapsing glomerulopathy, and protein leakage in Bowman's capsule $(8,9)$.

Another potential mechanism of AKI involves SARSCoV-2-related immune response dysregulation, as indicated by observed lymphopenia and cytokine release syndrome (cytokine storm) $(10,11)$.

Other contributors to AKI might include rhabdomyolysis, macrophage activation syndrome, and the development of microemboli and microthrombi in the context of hypercoagulability and endotheliitis $(7,12)$.

Maesaka et al (13) identified the various causes of hyponatremia by determining FEurate to propose an algorithm to approach hyponatremic conditions. In this algorithm, conditions with low FEurate $<4 \%$ include extrarenal salt losses with normal kidney function, Addison's disease, and edematous states such as heart failure, cirrhosis, and nephrosis. Normal FEurate of 4\%-11\% include psychogenic polydipsia and reset osmostat (RO), and increased FEurate $>11 \%$ SIADH/hydrochlorothiazide and C/RSW. SIADH can be differentiated from C/RSW by correcting the hyponatremia by any possible means and observing normalization of FEurate in SIADH as compared to being persistently increased in C/RSW. This algorithm greatly simplifies the diagnosis of patients with Addison's disease and RO.

The report of C/RSW occurring in patients without cerebral disease has led to proposal to change CSW to RSW (13). Maesaka et al (13) utilized this algorithm and differences in physiologic response to isotonic saline infusions between SIADH and RSW to evaluate hyponatremic patients from the general medical wards of the hospital. Isotonic saline does not induce excretion of dilute urines or correct the hyponatremia in SIADH as compared to inducing excretion of dilute urines and correction of the hyponatremia in RSW.

In the absence of cerebral disease and by exclusion of other reported causes that may be cause RSW as chemotherapy with cisplatin, myeloproliferative disorders, and post-operative fracture $(13,16)$ and as our patient is positive for COVID-19, hence we suggest that COVID-19 is the cause of RSW by affecting proximal kidney tubule transport through SARS-CoV-2 which can directly infect the renal tubular epithelium and podocytes through an angiotensin-converting enzyme 2 (ACE2)-dependent pathway (8.9) and other different mechanism involves SARS-CoV-2-related immune response dysregulation, as indicated by observed lymphopenia and cytokine release syndrome (cytokine storm) $(10,11)$.

\section{Conclusion}

In conclusion, RSW should be considered in patients with COVID -19 with hyponatremia and absence of cerebral disease. We suggest changing CSW to the more appropriate term RSW.

\section{Acknowledgments}

Many thanks to my colleagues in the Al Khezam dialysis center and Al Dabous cardiology center, Al-Adan hospital for following and collection of all data about the patient.

Authors' contribution

All authors contributed equally to prepare the manuscript.

Conflicts of interest

The authors declared that they have no conflict of interest.

Ethical considerations

Ethical issues (including plagiarism, data fabrication, double publication) have been completely observed by the authors.

Funding/Support

None.

\section{References}

1. Peters JP, Welt LG, Sims EA, Orloff J, Needham J. A salt-wasting syndrome associated with cerebral disease. Trans Assoc Am Physicians. 1950;63:57-64.

2. Tenny S, Thorell W. Cerebral Salt Wasting Syndrome. Treasure Island (FL): StatPearls Publishing; 2020.

3. Bettinelli A, Longoni L, Tammaro F, Fare PB, Garzoni L, Bianchetti MG. Renal salt-wasting syndrome in children with intracranial disorders. Pediatr Nephrol. 2012;27:733-9.

4. Moritz ML. Syndrome of inappropriate antidiuresis and cerebral salt wasting syndrome: are they different and does it matter? Pediatr Nephrol. 2012;27(5):689-93.

5. Maesaka JK, Imbriano LJ, Ali NM, Ilamathi E. Is it cerebral or renal salt wasting? Kidney Int. 2009;76(9):934-8.

6. Ronco C, Bellomo R. Kellum JA . Acute kidney injury. Lancet. 2019;394:1949-64.

7. Varga Z, Flammer AJ, Steiger P, Haberecker M, Andermatt $\mathrm{R}$, Zinkernagel $\mathrm{AS}$, et al. Endothelial cell infection and endotheliitis in COVID-19. Lancet. 2020;395:1417-8. doi: 10.1016/S0140-6736(20)30937-5.

8. Su H, Yang M, Wan C, Yi LX, Tang F, Zhu HY, et al. Renal histopathological analysis of 26 postmortem findings of patients with COVID-19 in China. Kidney Int. 2020;98(1):21927. doi: 10.1016/j.kint.2020.04.003.

9. Larsen CP, Bourne TD, Wilson JD, Saqqa O, Sharshir MA. Collapsing Glomerulopathy in a Patient With Coronavirus Disease 2019 (COVID-19) . Kidney Int Rep. 2020;5:935-9. doi:10.1016/j.ekir.2020.04.002.

10. Zhou F, Yu T, Du R, Fan G, Liu Y, Liu Z, et al. Clinical course and risk factors for mortality of adult inpatients with COVID-19 in Wuhan, China: a retrospective cohort study. Lancet. 
2020;395:1054-62. doi: 10.1016/S0140-6736(20)30566-3.

11. Ronco C, Reis T. Kidney involvement in COVID-19 and rationale for extracorporeal therapies. Nat Rev Nephrol. 2020;16:308-310. doi: 10.1038/s41581-020-0284-7.

12. Zhang $\mathrm{Y}$, Xiao M, Zhang S, Xia P, Cao W, Jiang W, et al. Coagulopathy and Antiphospholipid Antibodies in Patients with Covid-19. N Engl J Med. 2020;382:e38. doi: 10.1056/ NEJMc2007575.

13. Maesaka JK, Imbriano LJ, Miyawaki N. High Prevalence of Renal Salt Wasting Without Cerebral Disease as Cause of Hyponatremia in General Medical Wards. Am J Med Sci. 2018;35615-22. doi: 10.1016/j.amjms.2018.03.020.14.
14. Rahman M, Friedman WA. Hyponatremia in neurosurgical patients: clinical guidelines development. Neurosurgery. 2009;65(5):925-35; discussion 935-6

15. Maesaka JK, Miyawaki N, Palaia T, Fishbane S, Durham $\mathrm{JH}$. Renal salt wasting without cerebral disease: diagnostic value of urate determinations in hyponatremia. Kidney Int. 200;71(8):822-6.

16. Bitew S, Imbriano L, Miyawaki N, Fishbane S, Maesaka JK. More on renal salt wasting without cerebral disease: response to saline infusion. Clin J Am Soc Nephrol. 2009;4(2):309-15. 